\title{
Pure Red Cell Aplasia Associated with Good's Syndrome Accompanied by Decreased Stem Cell Factor Production in the Bone Marrow
}

\author{
Kageaki Kuribayashi ${ }^{1}$, Akihito Fujimi ${ }^{2}$, Masayoshi Kobune ${ }^{2}$, Rishu Takimoto ${ }^{2}$, \\ Shohei Kikuchi ${ }^{2}$, Satoshi Iyama ${ }^{2}$, Junji Kato ${ }^{2}$, Yoshiro Niitsu ${ }^{2}$ and Naoki Watanabe ${ }^{1}$
}

\begin{abstract}
A 79-year-old Japanese woman diagnosed with pure red cell aplasia (PRCA) associated with thymoma and hypogammaglobulinemia (Good's syndrome) was successfully treated with cyclosporine-A after a thymectomy. We further studied the etiology of this case. A burst-forming unit erythroid (BFU-E) assay with SCF restored erythropoiesis in vitro. SCF production was reduced in bone marrow stromal cells; however, it was restored in vitro and in vivo after cyclosporine-A treatment.
\end{abstract}

Key words: pure red cell aplasia, stem cell factor, thymoma, Good's syndrome, hypogammaglobulinemia, cyclosporine-A

(Inter Med 49: 377-382, 2010)

(DOI: 10.2169/internalmedicine.49.2811)

\section{Introduction}

Pure red cell aplasia (PRCA) is characterized by normocytic anemia, reticulocytopenia, and severe erythroid hypoplasia in the bone marrow without leukopenia or thrombocytopenia. This disorder may be congenital (DiamondBlackfan anemia) or acquired as an acute or chronic syndrome. Acquired PRCA has multiple pathogeneses, including the presence of serum inhibitors against erythroid precursors or erythropoietin (EPO), cellular inhibition of erythropoiesis, consumption of certain drugs, and chronic parvovirus B19 infection (1). Chronic PRCA is an uncommon disorder; the prevalence is reported to be 0.7 case $/ \mathrm{mil}-$ lion/year (2).

Thymoma associates with a variety of disorders including myasthenia gravis, PRCA, and hypogammaglobulinemia; the incidences are reported to be $15 \%, 5 \%$, and $5 \%$, respectively $(3,4)$. Hypogammaglobulinemia associated with thymoma is referred to as Good's syndrome (5). Complication of PRCA and Good's syndrome is extremely rare and only 12 cases have been reported to date (6).
Here, we report a case of PRCA associated with Good's syndrome, wherein hypogammaglobulinemia and PRCA were successfully treated with cyclosporine-A after a thymectomy. Furthermore, a low level of stem cell factor (SCF) production was detected, which recovered after the initiation of cyclosporine-A administration.

\section{Materials and Methods}

\section{Preparation of stromal cells}

Stromal cells were obtained according to a method described in a previous report (7). After informed consent was obtained from the present patient and a normal volunteer, bone marrow was collected by aspiration from the posterior iliac crest. Bone marrow mononuclear cells (BMMNCs) were plated in $75-\mathrm{cm}^{2}$ plastic tissue culture flasks and incubated overnight. After removing non-adherent cells, adherent cells were cultured in a long-term culture medium consisting of minimum essential medium supplemented with $12.5 \%$ horse serum (Gibco BRL, Rockville, MD), $12.5 \%$ fetal calf serum (Gibco BRL), $1 \mu \mathrm{M}$ hydrocortisone (Sigma, St.

${ }^{1}$ Department of Clinical Laboratory Medicine, Sapporo Medical University School of Medicine, Sapporo and ${ }^{2}$ The Fourth Department of Internal Medicine, Sapporo Medical University School of Medicine, Sapporo

Received for publication August 23, 2009; Accepted for publication November 13, 2009

Correspondence to Dr. Naoki Watanabe, watanabn@sapmed.ac.jp 
Louis, MO), and $100 \mu \mathrm{M} \beta$-mercaptoethanol (Sigma) at 37 ${ }^{\circ} \mathrm{C}$ in a humidified atmosphere containing $5 \% \mathrm{CO}_{2}$. Subconfluent cells were harvested and used as stromal cells for further experimentation.

\section{Burst-forming unit-erythroid colony assay}

A burst-forming unit-erythroid (BFU-E) colony assay was carried out as according to a method described in a previous report (8). Briefly, $1 \times 10^{6} \mathrm{BMMNCs}$ were plated on $35-\mathrm{mm}$ tissue culture dishes (Becton Dickinson, Franklin Lakes, NJ) containing $2 \mathrm{~mL}$ of a methylcellulose-based semisolid culture medium (MethoCult SF ${ }^{\text {BIT }}$ H4436; Stem Cell Technologies, Vancouver, Canada) with either $10 \%$ serum from a PRCA patient or normal individual. The patient and the control subject had the same red blood cell type (i.e., AB). Each test was performed in triplicate. After $14 \mathrm{~d}$ of incubation at $37^{\circ} \mathrm{C}$ in a $5 \% \mathrm{CO}_{2}$ atmosphere, BFU-E colonies were counted using an inverted microscope. The results are expressed as the mean values from 3 experiments. Statistical significance was determined using Student's t test.

\section{Semi-quantitative reverse transcriptase-polymerase chain reaction analysis of cytokine $m R N A$}

Reverse transcriptase-polymerase chain reaction (RT-PCR) analysis was performed for SCF, IL-3, and glyceraldehyde3-phosphate dehydrogenase (GAPDH) in stromal cells according to a method described in a previous report (7). Total RNA was extracted using the QIAGEN RNeasy kit (Qiagen, Tokyo, Japan). RT-PCR was performed with $1 \mu \mathrm{g}$ of total RNA using the GeneAmp RNA PCR kit (Applied Biosystems, Foster City, CA). For human SCF, 5'-GCC GCT GTT CGT GCA ATA TGC-3' was used as the forward primer and 5'-GGA GTA AAG AGC CTG GGT TCT GGG-3' was used as the reverse primer. For human IL3, 5'-GCT CCC ATG ACC CAG ACA ACG TCC-3' was used as the forward primer and 5'-CAG ATA GAA CGT CAG TTT CCT CCG-3' was used as the reverse primer. The reaction mixtures were subjected to PCR under the following conditions; $94^{\circ} \mathrm{C}$ for 30 seconds, $56^{\circ} \mathrm{C}$ for 30 seconds, and $72^{\circ} \mathrm{C}$ for 60 seconds.

\section{Quantification of soluble- and transmembrane-SCF}

Human SCF has 2 transcriptional variants: a soluble form and a transmembrane form. The expression of these mRNAs was determined by quantitative RT-PCR using the ABI PRISM 7700 sequence detection system (Applied Biosystems, Foster City, CA). The primers specific to the transcriptional variants and the fluorescent hybridization probes for quantitative RT-PCR were as follows. For soluble SCF, 5'TAG TGA TTG TGT GGT TTC TTC AAC ATT-3' and 5'TAA GGG AGC TGG CTG CAA CA-3' were used as the forward and reverse primers, respectively, and 5'CAG AGT CAG TGT CAC AAA ACC ATT TAT GTT-3' was used as the taqman probe. For transmembrane-SCF (TM-SCF), 5'AAT AGA TCC ATT GAT GCC TTC AAG GA-3', 5' '-TTT TTG GCC TTC CCT TTC TCA-3', and 5'-TAG TGA TTG
TGT GGT TTC TTC AAC A-3' were used as the forward primer, reverse primer, and taqman probe, respectively. The mRNA levels were normalized by calculating the ratio to that of $18 \mathrm{~S}$ ribosomal RNA (rRNA). The 18S ribosomal RNA was quantified using the TaqMan Ribosomal RNA control reagents (Applied Biosystems).

\section{Measurement of serum SCF level}

The serum SCF level was measured by the AN'ALYZA Human SCF immunoassay (TECHNE Corporation, Minneapolis, $\mathrm{MN}$ ) according to the manufacturer's instructions.

\section{Case Report}

A 79-year-old Japanese woman, who had been experiencing general malaise since September 2003, was diagnosed with macrocytic anemia (red blood cell (RBC), $1.06 \times 10^{6} / \mu \mathrm{L}$; hemoglobin $(\mathrm{Hb}) 3.7 \mathrm{~g} / \mathrm{dL}$; hematocrit (Hct) 11.0\%; mean corpuscular volume (MCV) 104; mean corpuscular hemoglobin (MCH) 34.9; and mean corpuscular hemoglobin concentration (MCHC) 33.6). She was referred to our hospital for further examination. In her history, 40 years previously, a mediastinal mass was detected. On admission, laboratory examination revealed anemia with reticulocytopenia $(4,240 /$ $\mu \mathrm{L})$ and the leukocytes and platelets counts were normal. The serum levels of iron, ferritin, and EPO were elevated; they were found to be $161 \mathrm{mg} / \mathrm{dL}, 274 \mathrm{mg} / \mathrm{dL}$, and 1,560 $\mathrm{IU} / \mathrm{mL}$, respectively. The results of direct and indirect Coombs tests were negative, and total bilirubin and lactate dehydrogenase levels were within the normal range. Due to hypogammaglobulinemia, the amount of total protein was low at $5.2 \mathrm{~g} / \mathrm{dL}$. Hepatitis B virus (HBV)-DNA was present without evidence of liver dysfunction. Hepatitis $\mathrm{C}$ virus (HCV) antibody, cytomegalovirus antigenemia and parvovirus B19 IgM antibody were absent. Viral capsid antigenimmunoglobulin $\mathrm{M}$ (VCA-IgM) and IgG-coated erythrocytes (EAIgG) were absent, and VCA-IgG and Epstein-Barr virus nuclear antigen (EBNA) were present. Examination of the bone marrow aspirate showed hypocellularity without dysplasia or the presence of leukemic cells. The myeloiderythroid ratio was 108:1, and cytogenetic analysis of the bone marrow revealed a normal karyotype. On the basis of these results, she was diagnosed with PRCA. A computed tomography scan of the chest showed a heterogeneous mass (size, $6 \times 7 \mathrm{~cm}$ ) in the anterior mediastinum. Thymectomy was performed in mid-December 2003. According to the World Health Organization (WHO) classification, the tumor was a type $\mathrm{A}$ thymoma with a type $\mathrm{AB}$ component. The cells did not display dysplastic features. Anemia and hypogammaglobulinemia persisted for 4 months after thymectomy; hence cyclosporine-A (300 mg/day) was administered. To prevent hepatitis B reactivation, administration of 100 $\mathrm{mg} /$ day lamivudine was initiated 2 weeks before the initiation of cyclosporine-A treatment. As shown in Fig. 1, the number of reticulocytes began to increase $14 \mathrm{~d}$ after cyclosporine-A treatment was initiated, and the hemoglobin 


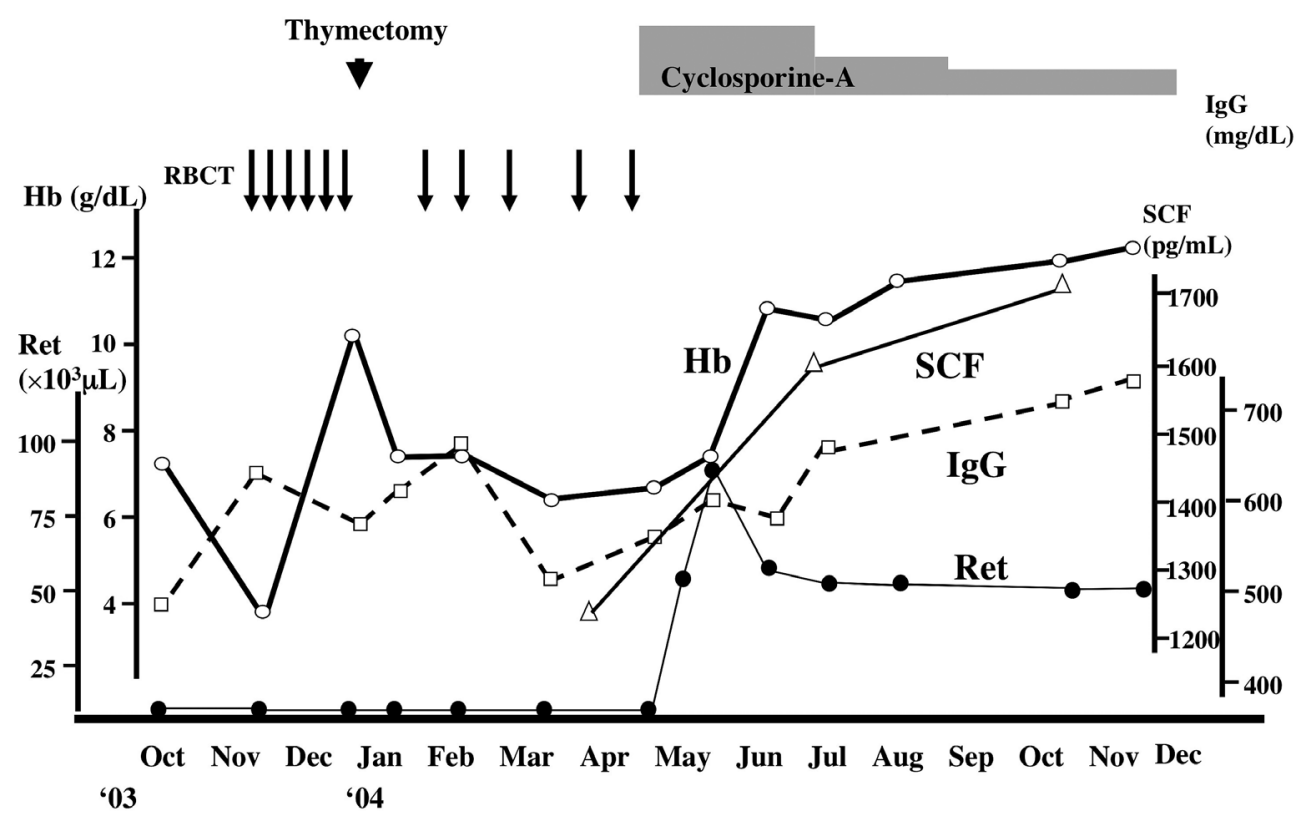

Figure 1. Clinical course of a case of PRCA associated with Good's syndrome. RBCT: red blood cell transfusion, $\mathrm{SCF}$ : stem cell factor $(\mathrm{pg} / \mathrm{mL})$, Ret: reticulocytes $\left(\times 10^{3} / \mu \mathrm{L}\right)$

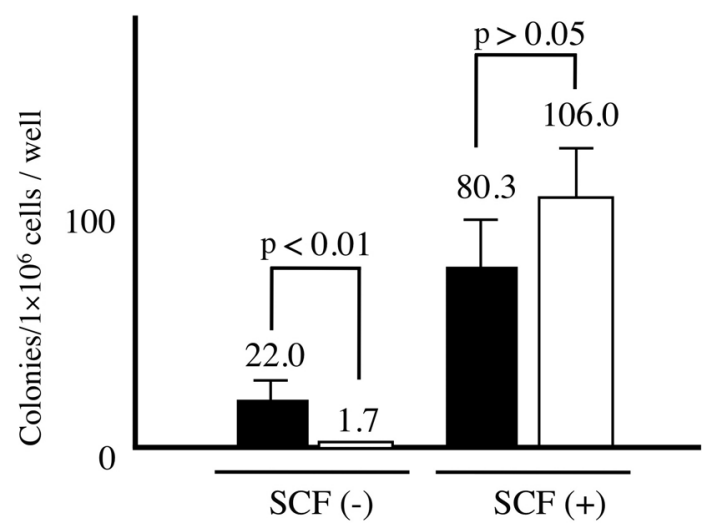

Figure 2. BFU-E colony assay. BFU-E assay was performed in the presence or absence of SCF. Closed bar, control BMMNCs; open bar, patients BMMNCs.

level improved to $10.4 \mathrm{~g} / \mathrm{dL}$ approximately 1 month after the treatment was initiated. The patient no longer required RBC transfusions. The IgG level also increased to $700 \mathrm{mg} /$ $\mathrm{dL}$ at 6 months after treatment.

To determine the etiology of PRCA in the patient, we first performed a BFU-E colony assay using the patient's BMMNC sample and the BMMNC sample of normal individuals. Contrary to our expectation, the number of BFU-E colonies produced by the patient's BMMNCs and that produced by the BMMNCs of a normal individual were similar (Fig. 2). The results were not affected when the assay was performed in the absence of T cells. Moreover, BFU-E colony formation was not inhibited when the patient's serum sample was used.

We conducted an in vitro experiment to determine why the patient's BMMNCs were able to form BFU-E colonies in vitro but not in vivo. Because Metho Cult (the medium used in BFU-E colony assay) contains recombinant human (rh) SCF, rh IL-3, and rh EPO, we hypothesized that one of these factors was lacking in our patient and that the addition of this missing factor would lead to the restoration of colony formation in in vitro. We obtained bone marrow stromal cells from the patient and a normal volunteer. Semiquantitative RT-PCR analysis of IL-3 and SCF was performed. To study the effect of cyclosporine-A on the cytokine production in stromal cells, stromal cells were cultured with or without $300 \mathrm{ng} / \mathrm{mL}$ cyclosporine-A for $7 \mathrm{~d}$. As shown in Fig. 3A, the SCF mRNA level was low in the patient's stromal cells as compared to that in the control cells; the SCF level was restored to normal levels after cyclosporine-A treatment was initiated. IL-3 was below the limit of detection in these bone marrow stromal cells. SCF has 2 transcriptional variants, i.e., the soluble and the transmembrane forms. We further quantified these mRNAs in bone marrow samples of the present patient and in those of 7 individuals without apparent disease in the bone marrow. As shown in Table 1, the levels of both SCF splice variants were low in the patient as compared to the controls, and the levels were restored with cyclosporine-A administration (Fig. 3B). From these results, we speculated that decreased SCF production in the bone marrow stromal cells was the cause of PRCA in this patient.

The serum protein levels of SCF were monitored before and during cyclosporine-A treatment. The SCF level increased after cyclosporine-A administration (Fig. 1); this result is consistent with in vitro RT-PCR results. Hb and gammaglobulin levels also increased with cyclosporine treatment. Before cyclosporine-A administration, CD4/CD8 was 1.10-1.56, which decreased to $0.60-0.75$ after the treatment.

Interestingly, the patient has never experienced lifethreatening infections throughout her clinical course. Ad- 

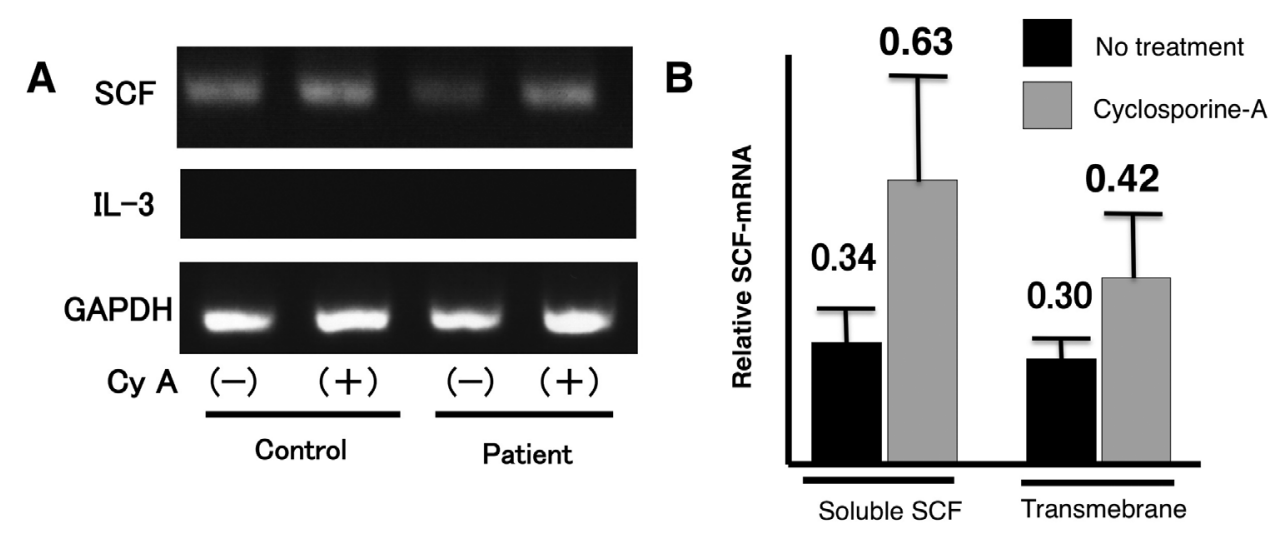

Figure 3. (A) RT-PCR analysis of bone marrow stromal cells, with or without cyclosporine-A treatment. SCF: stem cell factor, IL-3: interleukin 3. (B) Quantitative RT-PCR analysis of solubleand transmembrane-SCF mRNA production of patient bone marrow stromal cells before and after cyclosporine-A treatment.

Table 1. Relative Expression Level of Soluble and Transmembrane SCF

\begin{tabular}{ccllcc}
\hline Case & Age & Sex & Diagnosis & Sol-SCF & TM-SCF \\
\hline 1 & 8 & M & Malignant Lymphoma & 4.9 & 9.9 \\
2 & 29 & M & Normal Volunteer & 3.3 & 10.0 \\
3 & 59 & M & Malignant Lymphoma & 3.5 & 11.2 \\
4 & 61 & F & Renal Anemia & 5.9 & 15.4 \\
5 & 72 & F & Reactive Eosinophilia & 2.8 & 14.6 \\
6 & 75 & M & Renal Anemia & 0.7 & 8.3 \\
7 & 80 & M & Malignant Lymphoma & 2.4 & 14.0 \\
Present case & & & & \\
\multicolumn{7}{r}{} & & & & \\
\end{tabular}

ministration of cyclosporine-A is maintained and the patient is healthy to date; anemia and hypogammaglobulinemia are maintained at good levels.

\section{Discussion}

In this study, we report a case of PRCA associated with Good's syndrome accompanied by decreased SCF expression in bone marrow stromal cells. Consistent with recent notion, anemia responded to cyclosporine-A but not to a thymectomy (9).

$\mathrm{SCF}$ is the ligand for c-kit and the mutant mice ( $\mathrm{Sl} / \mathrm{Sl}{ }^{d}$ and $W / W^{\nu}$, respectively) display a severe anemic phenotype. It has been shown that SCF administration reverses the anemic phenotype in $S l / S l^{d}$ mice (10). As these mice models resemble human PRCA, extensive studies have been performed in order to detect a genetic alteration in c-kit or in the ligand in patients with congenital PRCA; however, these studies have been unsuccessful.

We detected low SCF production in bone marrow stromal cells in the present case. We speculate that this was the cause of PRCA in the present patient, because the addition of SCF restored the formation of BFU-E colonies in vitro, and cyclosporine-A treatment also increased SCF production in vivo and in vitro; this finding correlated with that observed in anemia recovery.

There are distinct differences in the biological characteristics of soluble SCF and TM-SCF. The expression levels of soluble SCF and TM-SCF differ among tissues (11), and TM-SCF is more effective than soluble form in terms of sustaining hematopoiesis (12). The $S l^{d}$ allele in $S l / S l^{d}$ mice generates SCF that lacks TM domain; the mice produce intact soluble SCF but not TM-SCF and leads to a macrocytic anemic phenotype $(11,13,14)$. Moreover, expression of transgenic TM-SCF restores erythropoiesis in $S l / S l^{d}$ mice although induced expression of soluble SCF does not restore erythropoiesis (15). In addition, Abkowitz et al have reported that the serum level of soluble SCF was neither elevated nor decreased in 34 patients with acquiredPRCA (16). Consistent with these reports, the serum level of soluble SCF in the present patient was within the reference range $(558-1,441 \mathrm{pg} / \mathrm{mL})$. From these observations, we speculate that not soluble SCF but the decreased expression level of TM-SCF in the bone marrow leads to the development of PRCA.

Because a $\mathrm{CpG}$ island was present in the promoter region of SCF, we performed methylation-specific PCR but found no patient-specific methylation of the region. The reason 
why the SCF expression level was decreased remains unknown.

It has been reported that SCF restores BFU-E colony formation in acquired and congenital PRCA $(17,18)$. Gussetis et al demonstrated that the response to SCF in the BFU-E assay is the predictive factor for the response to immunosuppressive therapy in PRCA patients (18). Hydrocortisone is known to stimulate SCF production in bone marrow fibroblasts (19); moreover, our study also showed that cyclosporine-A induces SCF production in bone marrow stromal cells. Thus, SCF may determine the response to immunosuppressive therapy in PRCA patients.

It is unknown how cyclosporine-A restored SCF expression. Cyclosporine-A inhibits the $\mathrm{Ca}^{2+} /$ calcineurin pathway and the subsequent activation of activation of the nuclear factor of activated $\mathrm{T}$ cell (NF-AT); this results in the suppression of early immune response, including T-cellmediated production of IL-2, IL-4, CD40, and the Fas ligand (20). SCF is constitutively produced by endothelial cells and fibroblasts; the production is modestly enhanced by IL-1 and tumor necrosis factor- $\alpha$ (TNF $\alpha)$, and suppressed by transforming growth factor- $\beta$ (TGF $\beta$ ) (15). It is likely that cyclosporine-A modulates the cytokine production profile in vivo, and this may have resulted in the increased SCF expression in bone marrow stromal cells.

In MG patients, an antibody directed against nicotinic acetylcholine receptors (nAchRs) induces symptoms associated with MG (21). Therefore, we have referred to the pathogenesis of myasthenia gravis (MG) associated with thymoma. However, our results did not indicate the presence of a serum inhibitor because the present patient's serum did not inhibit colony formation in the BFU-E or red blood cell production using two-phase system (data not shown) (22).
It has been shown that PRCA is associated with T-cell clonal disorders $(23,24)$. Therefore, we used $\mathrm{T}$ cells obtained from peripheral blood and the thymoma and preformed PCR southern analysis to analyze the rearrangement in the genes of T-cell receptor $\beta$ and $\delta$ chains; however, no clonality was found. We performed a colony assay in the absence of $\mathrm{T}$ cells and found that the number of BFU-E colonies was similar to that for sample obtained from normal volunteers. We were unable to detect erythropoiesis inhibitory T-cells in this patient.

A thymectomy was carried out in the present case not to improve anemia and hypogammaglobulinemia but to diagnose and treat the thymoma, as it was depressing the cardiac function of the heart due to its mass effect. However, thymectomy should be performed carefully in patients with Good's syndrome as it does not improve hypogammaglobulinemia and severe life-threatening infections might succeed after a thymectomy.

Taniguchi et al summarized that type-AB thymoma is the most frequent histology of thymoma which is associated with Good's syndrome and PRCA (6). Consistent with this report, the histology of the thymoma in the present case had type $\mathrm{AB}$ component.

In the case of our patient, the pathogenesis of hypogammaglobulinemia remains unclear. Both anemia and hypogammaglobulinemia were managed by cyclosporine-A administration, but not by thymectomy. This clinical course suggests that there is a common pathogenesis of PRCA and hypogammaglobulinemia. Because SCF is known to be required for the development and maintenance of lymphocytes (25), decreased SCF may be the reason for hypogammaglobulinemia.

\section{References}

1. Fisch P, Handgretinger R, Schaefer HE. Pure red cell aplasia. Br J Haematol 111: 1010-1022, 2000.

2. Sawada K. [Pure red cell aplasia: clinical manifestations and treatment]. Rinsho Ketsueki 47: 1438-1445, 2006 (in Japanese).

3. Thomas CR, Wright CD, Loehrer PJ. Thymoma: state of the art. J Clin Oncol 17: 2280-2289, 1999.

4. Rosenow EC 3rd, Hurley BT. Disorders of the thymus. A review. Arch Intern Med 144: 763-770, 1984.

5. Kelleher P, Misbah SA. What is Good's syndrome? Immunological abnormalities in patients with thymoma. J Clin Pathol 56: 1216, 2003.

6. Taniguchi T, Usami N, Kawaguchi K, Yokoi K. Good syndrome accompanied by pure red cell aplasia. Interact Cardiovasc Thorac Surg 9: 750-752, 2009.

7. Kawano Y, Kobune M, Yamaguchi M, et al. Ex vivo expansion of human umbilical cord hematopoietic progenitor cells using a coculture system with human telomerase catalytic subunit (hTERT)-transfected human stromal cells. Blood 101: 532-540, 2003.

8. Rappold I, Ziegler BL, Kohler I, et al. Functional and phenotypic characterization of cord blood and bone marrow subsets expressing FLT3 (CD135) receptor tyrosine kinase. Blood 90: 111-125, 1997.
9. Sawada K, Fujishima N, Hirokawa M. Acquired pure red cell aplasia: updated review of treatment. Br J Haematol 142: 505-514, 2008.

10. Zsebo KM, Williams DA, Geissler EN, et al. Stem cell factor is encoded at the Sl locus of the mouse and is the ligand for the ckit tyrosine kinase receptor. Cell 63: 213-224, 1990.

11. Huang EJ, Nocka KH, Buck J, Besmer P. Differential expression and processing of two cell associated forms of the kit-ligand: KL1 and KL-2. Mol Biol Cell 3: 349-362, 1992.

12. Toksoz D, Zsebo KM, Smith KA, et al. Support of human hematopoiesis in long-term bone marrow cultures by murine stromal cells selectively expressing the membrane-bound and secreted forms of the human homolog of the steel gene product, stem cell factor. Proc Natl Acad Sci U S A 89: 7350-7354, 1992.

13. Brannan CI, Lyman SD, Williams DE, et al. Steel-Dickie mutation encodes a c-kit ligand lacking transmembrane and cytoplasmic domains. Proc Natl Acad Sci U S A 88: 4671-4674, 1991.

14. Flanagan JG, Chan DC, Leder P. Transmembrane form of the kit ligand growth factor is determined by alternative splicing and is missing in the Sld mutant. Cell 64: 1025-1035, 1991.

15. Broudy VC. Stem cell factor and hematopoiesis. Blood 90: 13451364, 1997.

16. Abkowitz JL, Hume H, Yancik SA, Bennett LG, Matsumoto AM. 
Stem cell factor serum levels may not be clinically relevant. Blood 87: 4017-4018, 1996.

17. Abkowitz JL, Sabo KM, Nakamoto B, et al. Diamond-blackfan anemia: in vitro response of erythroid progenitors to the ligand for c-kit. Blood 78: 2198-2202, 1991.

18. Gussetis ES, Peristeri J, Kitra V, Liakopoulou T, Kattamis A, Graphakos S. Clinical value of bone marrow cultures in childhood pure red cell aplasia. J Pediatr Hematol Oncol 20: 120-124, 1998.

19. Linenberger ML, Jacobson FW, Bennett LG, Broudy VC, Martin FH, Abkowitz JL. Stem cell factor production by human marrow stromal fibroblasts. Exp Hematol 23: 1104-1114, 1995.

20. Beals CR, Clipstone NA, Ho SN, Crabtree GR. Nuclear localization of NF-ATc by a calcineurin-dependent, cyclosporin-sensitive intramolecular interaction. Genes Dev 11: 824-834, 1997.

21. Vincent A, Palace J, Hilton-Jones D. Myasthenia gravis. Lancet 357: 2122-2128, 2001

22. Fujimi A, Matsunaga T, Kobune M, et al. Ex vivo large-scale generation of human red blood cells from cord blood CD34+ cells by co-culturing with macrophages. Int J Hematol 87: 339-350, 2008.

23. Masuda M, Teramura M, Matsuda A, et al. Clonal $\mathrm{T}$ cells of pure red-cell aplasia. Am J Hematol 79: 332-333, 2005.

24. Rose MG, Berliner N. T-cell large granular lymphocyte leukemia and related disorders. Oncologist 9: 247-258, 2004.

25. Kang J, Der SD. Cytokine functions in the formative stages of a lymphocyte's life. Curr Opin Immunol 16: 180-190, 2004.

(C) 2010 The Japanese Society of Internal Medicine http://www.naika.or.jp/imindex.html 